\title{
Les régulations professionnelles déontologiques de communication publique : des valeurs et des normes de professionnalisation vecteurs de reconnaissance
}

\author{
Dominique Bessières \\ Maître de Conférences en Sciences de \\ I'Information et de la Communication \\ Université Rennes 2 - PREFICS EA 7469 \\ dominique.bessieres@univ-rennes2.fr
}




\title{
Résumé
}

Les régulations professionnelles au travers d'un certain nombre d'opérations éthiques et déontologiques visent à organiser une représentation d'unité d'un positionnement des métiers de communicateur public, comme les chartes et récemment un manifeste pour la communication publique. Des valeurs et des normes sont ainsi affichées, mais dans quelle mesure participent-elles d'un mouvement de professionnalisation interactionniste pour consolider le rôle, la fonction, vis-à-vis d'autres groupes sociaux ? Ainsi, plus largement, leur portée ne se résume pas à leur seul contenu, mais bien plus globalement à leur insertion dans des dynamiques de revendication sociale. Il ne s'agit pas de règles contraignantes en termes juridiques, aussi leur sens et leurs incidences sont plus globales. Elles participent, avec d'autres, à la recherche d'une définition de l'exercice du métier de communicateur public. Le rôle des associations professionnelles est central, d'autant plus que l'on peut noter certaines évolutions dans le temps, avec toujours une représentation d'une éthique publique.

Mots clés: Communication publique, déontologie, charte, management, professionnalisation

\begin{abstract}
Professional regulations through a certain number of ethical and deontological operations aim to organize a representation of unity of the positioning of public communicator professions, such as charters and recently a manifesto for public communication. Values and norms are thus displayed, but to what extent do they participate in an interactionist professionalization movement to consolidate the role, the function, vis-à-vis other social groups? Thus, more broadly, their scope is not limited to their content alone, but more broadly to their inclusion in social advocacy dynamics. These are not binding rules in legal terms, so their meaning and their implications are more global. They participate, along with others, in the search for a definition of the exercise of the profession of public communicator. The role of professional associations is central, especially since we can note certain changes over time, always with a representation of public ethics.

Keywords : Public communication, ethics, charter, management, professionalization
\end{abstract}


La professionnalisation des fonctions de communication est relativement récente. Elle pose en filigrane la question des enjeux de l'existence d'un groupe professionnel qui peut être appréhendé au travers de questions éthiques ou déontologiques. Dans un cadre professionnel, les deux acceptions peuvent apparaître largement synonymes, ce qui est à relier à leurs racines étymologiques. L'origine grecque du premier terme, "ethos", renvoie à l'idée de mœurs ou coutume. Aujourd'hui, le terme réfère davantage à l'idée de règles de conduite. L'éthique ne serait donc pas vérité intangible, à l'instar de la morale, mais davantage redevable d'un questionnement, de la recherche d'un équilibre, et à ce titre contingente. La déontologie étymologiquement issue du grec «deontos » réfère à l'idée de devoir. Aussi, elle impliquerait l'idée de droits et de devoirs liés à l'exercice d'une profession. Elle suggère l'idée de réglementer, d'organiser une profession. Ces détours étymologiques invitent à porter une attention particulière aux enjeux sociaux de définition, en ce que les processus de normalisation et de codification déontologiques participent à la production d'une identité professionnelle, et ce faisant, ils concourent à sa stabilisation. C'est plus précisément ce que nous voulons montrer vis à vis du champ de la communication publique. Les responsables de communication institutionnelle publique gèrent les services de communication, des prestataires (agences de communication...). Ils définissent les plans et les actions de communication.

Dès lors, une éthique professionnelle correspond à des valeurs (axiologie) et à des normes définissant des obligations concrètes (déontologie). Mais, les juristes apprennent qu'une distinction est à opérer entre le droit dit "positif», qui est la règle édictée par l'autorité juridiquement compétente; et le droit dit «naturel », qui indépendant d'une intervention de règle positive, peut en conséquence être produit par des pairs. Or, la professionnalisation s'entend le plus souvent dans les contextes contemporains sur le modèle des professions libérales. Ceci induit une régulation fondée sur une capacité d'auto-organisation et d'élaboration collective des valeurs et des normes professionnelles. Ainsi les normes et valeurs professionnelles, en matière de communication publique, appartiennent à ce que les juristes anglo-saxons appellent "soft law». C'est-à-dire précisément des normes issues de processus d'autorégulation entre pairs qui n'ont pas la valeur coercitive de textes juridiques. Pour autant, nous pensons que l'on ne peut pas totalement écarter le droit ou « hard law » en ce qu'il contribue, lui aussi, à la définition des identités professionnelles et qu'il conditionne puissamment les logiques de professionnalisation. En sorte que des éléments macrosociaux issus de l'action de l'Etat (droit, université, référentiels officiels de métiers) d'une part, s'articulent dans le renforcement du groupe professionnel, conjointement à une échelle microsociale (déontologies, associations) d'autre part.

Déjà, lors du 17ème Congrès de la SFSIC à Dijon en 2010 (Bessières, 2010), nous avions axé notre communication sur le fait que si les fonctions de communication 
institutionnelle représentent un ensemble de métiers abordé par les SIC, leur analyse doit être plus fouillée dans le champ public. En effet, les communicateurs publics ne peuvent pleinement intégrer la professionnalisation_par concours dominante dans le secteur public. Aussi, les communicateurs publics ${ }^{1}$, notamment les cadres, restent majoritairement des contractuels. En sorte que, collectivement, ils recherchent des stratégies alternatives de professionnalisation calquées sur celles du secteur privé au travers de régulations professionnelles éthiques et déontologiques. Cette dynamique d'affirmation de reconnaissance et de légitimation en groupe professionnel des communicateurs, existe, même si les outils sont perçus comme évolutifs et non stabilisés depuis les années 1980. La communication publique est une pratique avant d'être objet de théorisation. Cette plasticité est un vecteur de pérennisation d'une activité progressivement institutionnalisée à l'extérieur et à l'intérieur des institutions publiques. On mesure un processus de construction d'une réalité qui devient objective dans des instruments, des services, des formations, des associations professionnelles, des chartes, tangibles. Aussi, comme le souligne Pierre Bourdieu (1982) "instituer, assigner une essence, une compétence, c'est imposer un droit d'être qui est un devoir être (ou d'être)." (....)"Deviens ce que tu es, telle est la formule qui sous-tend la magie performative de tous les actes d'institution ». Bien plus, la communication publique tend à devenir une discipline entendue comme la conjonction des savoirs professionnels et universitaires labellisant un secteur professionnel (Bessières 2009 a).

La communication représente un cadre propice d'analyse pour comprendre l'articulation du « social » et du « symbolique » (Bouillon, Bourdin, Loneux, 2008), en particulier des interactions sociales dans un cadre collectif large, vecteur de reconnaissance pour les acteurs de la communication publique. Nous avions noté (Bessières, 2010) que le terme de professionnalisation des fonctions de communication institutionnelle a été employé par des auteurs SIC dans les années 1990 ; il apparaissait nécessaire d'en préciser et d'en renforcer le concept, sous l'effet en particulier de l'émergence du développement de formations dédiées et de référentiels de compétences officiels récents. La recherche de la reconnaissance s'inscrit entre deux pôles, entre une culture métier et une culture des organisations publiques.

Une des difficultés pour étudier la thématique de l'éthique professionnelle réside dans le fait qu'elle regroupe une pluralité d'expertises académiques. Notre démarche compréhensive mobilise une grille pluridisciplinaire avec des éclairages théoriques issus des SIC, de la sociologie des professions, de la science politique, des sciences de gestion. Notre analyse intègre des éléments d'observation depuis une vingtaine d'années de manifestations organisées par des groupements d'acteurs, et

\footnotetext{
${ }^{1}$ On trouve aussi l'appellation de communicants.
} 
particulièrement une bonne connaissance directe des enjeux portés par l'association Communication publique ${ }^{2}(300$ membres, principale association sur ce secteur en France). Elle repose également sur des entretiens longitudinaux d'acteurs depuis $1993^{3}$. L'observation participante (Chapoulie, 1984) permet d'avoir accès à des niveaux d'informations difficilement appréhendables pour quelqu'un d'extérieur. En raison de notre statut d'universitaire nous n'appartenons pas au groupe professionnel des communicateurs publics. Aussi une distanciation est aisée vis à vis des enjeux strictement socio-professionnels du groupe social. Constituée en 1989, l'association est de loin la plus importante dans le champ professionnel ${ }^{4}$. Elle regroupe des directeurs de la communication importants de l'Etat, des collectivités locales, des institutions publiques et parapubliques, des entreprises publiques. L'association professionnelle délivre un discours, des valeurs proclamés et proposés pour références identitaires, à destination des membres du réseau professionnel en particulier, mais également vis-à-vis d'autres groupes sociaux. En cela c'est un lieu d'échange et de lobbying. Des principes déontologiques sont ainsi formalisés. Nous focaliserons notre analyse plus particulièrement sur deux documents qui ont été élaborés par l'association Communication publique, à savoir une Charte de 1998 et plus récemment un Manifeste en 2015.

L'existence de chartes déontologiques pour les communicateurs publics met en exergue des questionnements de nature éthique sur leurs pratiques, mais leur sens doit être compris dans une perspective large vis-à-vis d'autres groupes d'acteurs sociaux. Il s'agit d'opérations d'affichage, d'affirmation d'un positionnement professionnel, de construction symbolique d'une image valorisante, rendant compte d'un certain consensus entre les pairs. Notre démarche, fondée sur l'étude des chartes déontologiques des associations de communicateurs publics et sur des observations participantes, vise donc à révéler leur complexité en tant que terrains significatifs, mais non exhaustifs, de luttes sociales de définition d'un groupe professionnel. En effet, elles visent à afficher une image d'un professionnalisme, mais leur portée ne peut se réduire à leur seule textualité, bien plus elle nécessite d'être réintégrées dans la complexité d'un jeu social pluri-acteurs et pluridimensionnel. Les acteurs d'associations professionnelles œuvrent, en partie par leur biais, à conforter un groupe professionnel vis-à-vis de lui-même et des autres groupes sociaux de son environnement. Un des intérêts de recherche réside dans la pluralité de destinataires visés possibles, tout en appréhendant la complexité de positionnement professionnel de la communication publique. Les chartes étudiées représentent des visions non exhaustives, des tentatives d'unification de

\footnotetext{
${ }^{2} \mathrm{http}: / /$ www.communication-publique.fr/

${ }^{3}$ Nous ne rentrerons donc pas dans le détail de nos champs de recherche et nous renverrons à nos publications pour cela.

${ }^{4}$ On pourrait citer des associations pour la communication locale (Fourdin, 1994).
} 
représentation du métier de communicateur public, lesquelles peuvent être amenées à évoluer dans le temps en raison de dynamiques sociales. Nous voulons ainsi ouvrir un champ de réflexion et d'analyse sur ces différents phénomènes et débats qui concernent les relations ardues entre professionnalisation, compétence, métier, légitimité, norme, reconnaissance, notamment sur les relations entre représentation et champs professionnels en communication organisationnelle. Quel sens peut-on donner à l'éthique et la déontologie en matière de communication publique et quel peut-être le rôle pour les associations professionnelles sur ces enjeux ?

\section{Une définition externe et interne de l'exercice du métier de communicateur public}

Une fonction relativement récente peut être appréhendée au travers d'une grille rendant compte du processus où les valeurs et principes qui participent à la définition d'exercice professionnel sont fortement influencés par des regroupements de professionnels.

\subsection{Le prisme de la professionnalisation : déontologie et éthique}

En France, la thématique de la professionnalisation sur les métiers de communication a longtemps été peu étudiée en sociologie (Gadéa, 2003). Historiquement la sociologie de la professionnalisation a été un parent pauvre de la sociologie, où durablement la sociologie du travail a été prépondérante. L'influence du Laboratoire Printemps, fondé en 1995 à l'Université Versailles Saint Quentin en Yvelines, et de son premier Directeur, le Professeur Pierre Dubois, a été importante ; sa réussite institutionnelle a été accompagnée par le développement d'une demande sociale plus ouverte sur ces questions. L'analyse du rôle de regroupements d'acteurs visant à la reconnaissance d'un champ social particulier comme profession est une composante de la sociologie des professionnalisations. Dans un registre similaire, dans le champ des Sciences de l'Information et de la Communication, la professionnalisation des acteurs de la communication des organisations a constitué longtemps un parent pauvre de la recherche à quelques exceptions près durant les années 1990 (voir les textes de Walter, Fourdin, Pailliart, Bessières).

Nous avons été un des premiers à avoir introduit un des apports de la sociologie de la professionnalisation sur le champ des métiers de la communication, en particulier l'importance de la «professionnalisation interactionniste» dans des 
communications de colloques dès $2007^{5}$, en termes de dynamisme d'affirmation du groupe professionnel de communicateur vis-à-vis d'autres groupes. Aujourd'hui, il n'en n'est plus de même: l'analyse de la professionnalisation des acteurs de la communication est assez bien représentée dans le champ des Sciences de l'Information et de la Communication depuis quelques années.

En France, les communicateurs publics n'ont pas été oubliés par la régulation étatique (Dubar, Tripier, Boussard, 2015). L'émergence, puis l'accroissement, des filières de formation universitaires spécifiques contribuent à faire surgir la question de l'identité corporatiste qui donne unité et sens à ce qui n'est au départ qu'une agrégation abstraite de rôles professionnels (Bessières 2012). Depuis une vingtaine d'années, à l'instar des métiers de la communication en général, nous pouvons constater la constitution d'un champ professionnel par le biais de la création de formations diplômantes en communication publique. Elles se sont développées et se sont organisées au sein des universités, des écoles spécialisées, des instituts d'études politiques (Bessières, 2009b) et même au sein de l'École Nationale pour l'Administration (Bessières, 2012) confirmant ainsi une reconnaissance par le monde de l'enseignement supérieur et par la haute fonction publique.

Les prescriptions légales constituent également un puissant support de renforcement de la professionnalisation. Le droit public français reconnait implicitement la communication publique ${ }^{6}$ ainsi intégrée dans la pratique des organisations (Duran 1992). La loi du 15 janvier 1990 interdit au moins six mois avant un scrutin national la personnalisation de la communication, la promotion publicitaire de la gestion, de nouvelles actions de communication. En éliminant des communications politiques ouvertement personnalisées des élus au profit d'une communication d'apparence publique pérenne (Bessières 1998), les règles de droit contribuent à renforcer le groupe professionnel. Leurs applications concrètes relèvent de l'expertise des professionnels de la communication, dont la présence est nécessaire pour éviter des risques de remise en cause des élections en cas de manquements. Plus largement, l'activité communicationnelle est régie par des normes impersonnelles juridiques qui la légitiment en définitive (Laufer, Paradeise 1982).

La structuration et l'organisation des champs professionnels de la communication s'avèrent centrales dans la reconnaissance des pratiques communicationnelles des organisations, leur consolidation et leur renforcement. Les notions de zone d'expertise (Crozier), de champs (Bourdieu), de groupe professionnel (Chapoulie, Dubar et Tripier) rendent compte des phénomènes de légitimation de la

\footnotetext{
${ }^{5}$ Cf. Bessières, 2007, 2008.

${ }^{6}$ À la différence de l'Italie, seul pays européen doté d'une loi régissant le champ (loi du 7 juin 2000 n. 150).
} 
professionnalisation d'un certain nombre d'acteurs sociaux spécialisés dans les actions de communication des organisations. Le renforcement, puis la pérennisation, enfin l'institutionnalisation d'une expertise professionnelle reconnue s'effectue au sein des organisations, entre des pairs, ou avec des autorités hiérarchiques, institutionnelles, universitaires (Bessières, 2012).

Ainsi, notre démarche de recherche vise à éclairer les enjeux de professionnalisation pour le groupe des communicateurs publics. Celle-ci peut viser la constitution d'une nouvelle profession, une socialisation socle d'un développement de carrière, la formation de professionnels par des instances d'enseignement ou encore un processus de légitimation de la spécialisation des fonctions professionnelles de communication; en ce sens, elle est un facteur d'identité professionnelle (Dubar, Tripier, Boussard, 2015). Des signes de professionnalisation et d'institutionnalisation des communicateurs publics ${ }^{7}$ montrent une recherche de stabilisation pour obtenir la reconnaissance d'un professionnalisme particulier.

\subsection{Les associations professionnelles acteurs premiers}

Le rôle des associations professionnelles s'avère central dans la réussite des opérations de professionnalisation. Elles ont pour fonction de promouvoir la reconnaissance sociale, en propageant des stéréotypes de la profession garantissant existence et unité dans la réalité sociale professionnelle (Chapoulie 1973).

Ces types d'organisations collectives agissent pour faire connaître et reconnaître des professionnalismes particuliers, ce qui est très important en matière de légitimité et de légitimation d'un certain nombre d'acteurs spécifiques. Les évolutions récentes des fonctions, des professions, des métiers des communicateurs concourent à des structurations des champs professionnels en dynamiques. Celles-ci peuvent être dans certains cas centrifuges, rendant plus délicats la question des frontières entre des regroupements professionnels établies dans des associations et organisations spécifiques, des alliances pour soutenir des enjeux communs. Ces mouvements peuvent aussi être centripètes, en visant des reconnaissances de sous-champs professionnels définis en fonction d'une compétence spécifique (relations presse, communication numérique, communication digitale, communication interne, relations extérieures, relations publiques et institutionnelles, communication de marque, gestion documentaire, audiovisuels...).

L'appellation "communication publique" regroupe une grande diversité de fonctions effectives, de profils professionnels (chargé de communication, de relation

${ }^{7}$ Constitutifs d'une branche particulière très peu analysée de la sociologie des professions de cadre (Gadéa, 2003). 
publique et presse, journaliste, communication digitale...), de diplômes (droit, science politique, sciences de gestion, communication...). Si bien que l'on ne trouve guère d'enjeux communs entre tous les communicateurs en dehors d'une quête constante de reconnaissance (Pailliart, 1993). Or, la profession suppose une forme d'encadrement du métier évacuant le flou ${ }^{8}$.

«La professionnalisation est un processus selon lequel un corps de métier tend à s'organiser sur le modèle des professions établies, c'est-à-dire, métiers qui ont développé un ensemble de caractéristiques spécifiques; monopole d'exercice de certaines fonctions, contrôle des praticiens par leurs pairs » (Chapoulie, 1973).

On comprend alors l'enjeu de la quête de professionnalisation (Bessières, 2009b).

L'organisation de la légitimation de la profession (entendue dans le sens anglais d' «occupation»), mais avec des éléments inspirés des professions établies (proche $\mathrm{du}$ sens anglais de "profession »), comme la déontologie, implique de comprendre la redéfinition des cadrages et des délimitations issus des univers professionnels, sources d'identités professionnelles. Ainsi des pratiques plus anciennes contestées par une majorité de professionnels sont révélatrices de légitimations identitaires reconnues comme constitutives de bonnes pratiques: comme la critique de la communication publicitaire "paillette» des années 1980, la communication commerciale trop simplificatrice de la complexité du service public (cf. Zémor, 2008) des années 1990, ou encore de «la com» formulation délégitimante partagée dans le champ professionnel visant une communication publicité et marketing portée sur des « coups de com », des mises en scène peu porteuses de sens, court-termistes, voire peu éthiques des années 2010 (cf. Habib, 2010) (Bessières, 2016).

$\mathrm{Au}$ travers de ces représentations exemplaires proposées au groupe professionnel des communicateurs publics, on perçoit bien qu'il s'agit d'une lutte destinée à forger des représentations performatives. Ces opérations de construction de valeurs et de représentations propres contribuent à définir une identité professionnelle spécifique au travers de dynamiques de reconnaissances sociales de certaines fonctions (Dubar, Tripier, Boussard, 2015), dont les associations de professionnels sont souvent acteurs en particulier en les formalisant.

\section{Communication publique : d'une charte à un manifeste}

Notre connaissance de l'association Communication publique depuis 1994 au cours de notre thèse de doctorat, puis notre position de membre de son conseil d'administration nous permettent de rendre compte d'actions fortes de l'association (Bessières, 2012, 2016). Elles ont en commun d'œuvrer au renforcement du champ

\footnotetext{
${ }^{8}$ C'est une spécialisation professionnelle au sens de Dubar et Tripier.
} 
professionnel de la communication publique avec un affichage de valeurs déontologiques plus affirmatif dans le temps.

\subsection{Une première charte non contraignante}

Tout d'abord, l'Association a produit une Charte en 1998 reproduite ci-dessous (tableau 1). Elle visait à mettre en avant «des principes d'actions et de règles de comportements pour les communicateurs publics », énonçant « quelques principes et procédures qui puissent servir de références opératoires », avec « un texte bref, dans la mesure où il est impossible de statuer sur toutes les situations auxquelles peuvent être confrontés les acteurs de la communication publique », selon son préambule.

\footnotetext{
La communication publique a pour finalités l'échange et la partage d'informations d'utilité publique ainsi que le maintien du lien social, dans les rôles de régulation, de protection et d'anticipation qui incombent aux pouvoirs et services publics.

La communication publique inclut toute communication effectuée par des agents travaillant dans des institutions publiques, c'est-à-dire les corps constitués, les administrations centrales et leurs services déconcentrés, les collectivités territoriales et leurs instances de coopérations ainsi que les entreprises ou établissements ayant des missions de service public ou par toute autre personne devant respecter un cahier des charges émanant d'une institution publique.

Toute personne exerçant un métier de communication dans le cadre d'une organisation publique est soumise à des obligations particulières : ses devoirs ne sont pas identiques à ceux d'une personne exerçant un métier de communication dans le cadre d'une organisation privée.

La communication publique est attachée à favoriser l'accès à l'information, à promouvoir la transparence, à améliorer la relation de service.

Elle doit aussi accompagner les actes et décisions publics dans leur préparation, leur annonce et leur mise en œuvre.

Principes d'actions

1 - La communication publique doit être au service de l'intérêt général tel qu'il est légalement défini. Elle doit être au service de l'institution ou de l'entreprise au nom de laquelle on communique dans la mesure où cette communication ne méconnaît pas l'intérêt général attaché à la mission de l'institution. Elle doit se garder d'être au service d'intérêts particuliers qui seraient contradictoires avec l'intérêt général. Les messages d'intérêt général ne sauraient être, dans leur expression, détournés à des fins particulières.

2 - Les communicateurs publics sont responsables à la fois devant l'autorité publique - l'organisation pour laquelle ils communiquent - et devant les citoyens ou toute personne concernée par la communication publique.
} 
3 - La communication publique doit considérer, dans ses démarches, ses destinataires comme des récepteurs actifs : des citoyens à part entière dont procède l'intérêt général ; des hommes et des femmes rationnels et raisonnables ; des personnes chacun et chacune en situation particulière.

4 - La communication publique doit rendre compte à chacun de l'état des informations et des motivations des décisions qui les concernent.

5 - La communication publique doit s'assurer : de la diffusion la plus large des décisions publiques afin de ne privilégier des destinataires particuliers que dans la mesure où ils contribueront à une meilleure information du public; de l'accès aux informations que les institutions sont tenues de mettre sur la place publique; de l'égalité de traitement des journalistes en fonction des caractéristiques de leurs médias et de leurs publics; de ne pas tromper ses destinataires par omission.

6 - La communication publique doit intervenir dès que possible et aux moments les plus adaptés pour la majorité des personnes concernées par l'élaboration et la prise des décisions dont elle doit faire part. Elle doit être mise en œuvre en amont des décisions ; les responsables de communication doivent, si nécessaire, intervenir dans ce sens auprès de leurs dirigeants. Elle doit favoriser l'explication de façon à réduire les incompréhensions, les litiges ou les conflits et éviter le recours aux sanctions.

7 - La communication publique doit favoriser les démarches et les procédures qui permettent à chacun des destinataires intéressés de s'exprimer à tout moment d'un processus de décision. Elle doit favoriser le dialogue à chaque fois qu'une procédure ou qu'un service offert au public peut être adapté ou ajusté à une demande. Elle doit favoriser le débat lorsqu'un choix est ouvert aux citoyens.

Règles de comportements

1 - Etre, particulièrement dans la conduite des opérations de communication, attentif aux dispositions légales qui préservent l'égalité des citoyens et assurent le bon usage de l'argent public engagé : éviter le mélanges des genres entre la communication institutionnelle (moyens, fonds, procédures publics) et la communication politique ; considérer la communication comme partie intégrante du service offert au public, c'est-à-dire incluse dans le fonctionnement de l'institution publique ; garantir des critères objectifs d'attribution dans les recours aux prestataires, notamment en fonction de leurs savoir-faire.

2 - Privilégier l'information et l'explication et ne pas céder aux seuls artifices de séduction.

3 - Rechercher la cohérence entre la communication institutionnelle externe et la communication interne, donc en particulier entre image donnée et identité vécue.

\section{TABLEAU 1}

Charte de l'Association de Communication Publique rédigée en 1998 
Il ressort qu'au travers de la Charte de déontologie, les principes généraux de définition de la communication publique et de son rôle au service de l'intérêt général sont affichés dans une transcription présentée comme incitative et non contraignante. La charte met ainsi en avant la communication publique comme œuvrant directement aux objectifs et finalités des organismes publics, en indiquant des éléments pour clarifier son rôle (principes d'action) et en incitant à un exercice professionnel déontologique (règles de comportement).

\subsection{Un manifeste pour une affirmation plus mobilisatrice}

Puis, en 2015 l'association a produit un nouveau positionnement déontologique, mais davantage focalisé sur la revendication et l'affirmation d'un positionnement professionnel vis-à-vis des dirigeants publics dans un Manifeste (http://www.communication-publique.fr/manifeste-pour-la-communicationpublique/) (cf. Bessières, 2016 pour plus de détails sur le processus). Dans le contexte de remise en cause de la parole politique dans les enquêtes d'opinion, des communicateurs publics de l'association ont souhaité affirmer leur intérêt pour lutter contre une crise de légitimité et de confiance, notamment pour se démarquer de pratiques professionnelles jugées en partie responsables (ex. «coups de com»). Il s'agit de réaffirmer l'intérêt de la communication pour les décideurs publics et des postures déontologiques professionnelles.

Les conceptions du manifeste peuvent être synthétisées autour de 5 idées principales pour promouvoir un cadrage de l'action de la communication publique :

1. dépasser une communication court termiste (idée d'une communication s'inscrivant dans la durée pour des enjeux ou des stratégies de long terme opposée à une communication essentiellement supplétive),

2. transmettre du sens (pour clarifier, justifier, expliquer l'action publique),

3. développer une écoute (pour mettre en place une proximité et une attention à l'égard des populations et des contextes des organisations publiques),

4. intégrer la communication dès la conception de l'action publique (pour revendiquer une position stratégique et éviter une communication instrumentale de ratification ou d'exécution de décisions déjà prises),

5. privilégier une sincérité dans le langage (pour éviter des communications de dissimulation ou de duplicité attentatoires aux logiques d'intérêt général).

Ces deux textes déontologiques à 17 ans d'intervalle attestent du caractère processuel des opérations de légitimation $\mathrm{du}$ groupe professionnel des communicateurs, des responsables et directeurs de la communication, dans une dimension interactionniste pour être reconnus vis à vis d'autres groupes professionnels, et notamment les décideurs publics. Avec l'évolution terminologique de « charte », signifiant une norme non contraignante, à «manifeste » référant une 
implication active de la part des professionnels, tout se passe comme si la professionnalisation durant cette temporalité avait permis une certaine autonomisation cristallisée dans une plus grande capacité de revendication, plus ouvertement affichée. On mesure également par l'intermédiaire de ces deux exemples, l'importance des écrits qui participent à une cristallisation d'un accord contingent scripturalement qui peut donc être changé au cours du temps, d'autant plus qu'ils expriment une représentation du groupe conforme à l'air du temps.

\section{Des représentations collectives importantes socialement}

Les enjeux de figuration de la communication sont importants à une échelle globale mais aussi dans le champ public.

\subsection{Des représentations éthiques importantes dans le champ de la communication}

La construction collective de la reconnaissance de la fonction de communication est métissée entre culture professionnelle et organisations publiques. Elle se manifeste par des ajouts successifs. Lesquels sont collectivement instrumentalisés dans une stratégie de renforcement des communicateurs publics. Les acteurs sont informés des modèles de groupes de communicateurs antérieurs («publicitaires» Neveu, 2006; "conseils en communication politique», Champagne, 1990). Derrière l'imitation, ils en appliquent les règles, mais on mesure que la légitimation du groupe est une condition d'intégration pérenne dans une structure de travail fonctionnarisée dans une professionnalisation ambivalente (entre modèles privé et public).

En conséquence, un code de déontologie est symboliquement important dans l'affirmation d'une profession, même non établie, à l'exemple de celui de la publicité. Cette opération est réalisée par l'intermédiaire de l'Autorité de régulation professionnelle (ARPP). Ainsi, l'industrie publicitaire s'autorégule avec des codes de déontologie, moins restrictifs, au lieu de se voir imposer des réglementations juridiques (Loneux, 2016). Un tel code organise une forme de reconnaissance sociale du groupe, de son expertise et de sa zone de pouvoir en établissant des normes professionnelles, en rationalisant des pratiques, en proposant une identité (Walter, 1995).

L'association «Communication Publique» s'en rapproche au travers de ces deux exemples de réflexion déontologique, aboutissant à une charte déontologique en 1998 et un manifeste en 2015. L'exercice est symbolique d'une vision que des acteurs tentent d'imposer. D'autres regroupements professionnels, plus spécialisés, notamment en matière de communication territoriale ont aussi produit des écrits 
déontologiques proposant quelques principes éthiques, comme la charte déontologique de Cap'com, dite " charte de Marseille », qui fut votée, lors de la première convention nationale des communicateurs publics en novembre 2002 (Le Saëc, 2012). Cette dernière met en avant que « devant le déploiement croissant de la société de l'information, la communication publique s'affirme aujourd'hui comme une nécessité incontournable des institutions et des états démocratiques ». Ainsi, cette charte a pour but de «tracer les contours de l'exercice de la communication locale de service public, des droits et devoirs des professionnels qui l'exercent » et "d'élaborer "un contrat public" entre les partenaires qui la pratiquent : décideurs, employeurs et communicants». Elle «doit s'exercer hors de toute propagande ou falsification des faits et respecter la nécessaire transparence des informations dont elle dispose, tant à l'intention des décideurs que des usagers ». Ainsi, la communication publique "s'inscrit dans le cadre des missions de l'information de service public et en respecte les règles en vigueur, tant déontologiques que juridiques ", "d'éviter le mélange des genres entre communications institutionnelle et politique » et de "privilégier l'information et l'explication aux seuls artifices de séduction ». L'étude des chartes déontologiques des associations de communicateurs publics révèle que ces professionnels partagent des valeurs. En l'occurrence, ils revendiquent une différenciation éthique par rapport à la communication politique professionnelle et un professionnalisme au moyen d'un positionnement spécifique vis à vis des dirigeants et des organisations. Tout se passe comme si ces chartes avaient vocation à établir une frontière d'ordre éthique entre les professionnels de la communication publique et ceux de la communication politique.

\subsection{Une représentation d'éthique publique importante pour les professionnels}

Le développement du métier de communicateur public symbolise des voies d'adaptation des organisations publiques aux contraintes extérieures de leur environnement social. Alors, le développement du métier doit être appréhendé comme une tentative de redéfinition des relations existantes entre l'État et les citoyens, car « l'argumentation (et la communication publique ont...) pour effet de tisser un lien réfléchi, d'établir une relation forte, de reconnaitre implicitement l'existence d'une obligation ; elle oblige un pouvoir à descendre de son piédestal » (Zémor, 2008). Nous pouvons ainsi constater que le développement du métier de communicateur public rend compte d'une évolution de la communication des organisations publiques, moins bureaucratique (axée sur le langage du droit et des résultats évalués en fonction de la conformité à des normes légales et administratives) de et plus axée sur des logiques managériales (basées sur le langage gestionnaire et une recherche de performance d'action par rapport à des objectifs et des mesures d'évaluations; Bessières, 2009a). Ces chartes éthiques revendiquent 
des valeurs professionnelles. Nous pouvons appréhender les créations de chartes déontologiques comme la concrétisation de dynamiques d'affirmation de valeurs partagées par ces professionnels et induisent le respect de certains comportements. Elles contribuent à forger la reconnaissance de leur singularité qui repose grandement sur la notion de service de l'intérêt général.

Plus précisément, les principes d'action et les règles contenus dans les chartes déontologiques des communicateurs publics tendent à préconiser l'adoption d'une conception positive des administrés pour lesquels les professionnels de la communication institutionnelle publique doivent "favoriser l'explication de façon à réduire les incompréhensions, les litiges ou les conflits et éviter le recours aux sanctions ». Ils doivent considérer "ses destinataires comme des récepteurs actifs : des citoyens à part entière dont procède l'intérêt général, des hommes et des femmes rationnels et raisonnables, des personnes chacun et chacune en situation particulière ". Aussi, "les messages ne sauraient être, dans leurs expressions, détournés à des fins particulières, [et] les communicateurs publics sont responsables à la fois devant l'autorité publique - l'organisation pour laquelle ils communiquent - et devant les citoyens » (Zémor, 2008).

Mais, bien que le métier de communicateur public soit présenté comme se définissant par rapport à la légitimité de l'intérêt général, il demeure que les professionnels de la communication publique sont liés au pouvoir politique et leurs rapports au politique ne doivent pas être occultés. Il apparaît que le métier de communicateur public reste dépendant du pouvoir politique auquel il est rattaché plus ou moins directement. Le changement des responsables de la communication à l'issue des échéances électorales le démontre le plus souvent. "La dépendance vis$\grave{a}$-vis du pouvoir politique reste manifeste » (Pailliart, 1993).

Les chartes déontologiques œuvrent au développement du métier de communicateur public, mais elles manifestent aussi intrinsèquement des revendications d'autonomisation vis-à-vis du pouvoir politique par l'établissement de frontières d'ordre éthique entre la communication publique et la communication politique. Cela se traduit par le partage de stéréotypes de bon professionnalisme critiquant comme contreproductive la centration excessive sur une personnalisation de la communication autour des discours et représentations des édiles par exemple, et plus encore par le respect des règles de la loi de 1990 qui limite l'usage à visée électoraliste de la communication des organismes publics comme cela pouvait être le cas auparavant.

Au total, la communication publique se définit par la légitimité de l'intérêt général et reste majoritairement à sens unique (pour plus de détails cf. Bessières, 2009a). L'existence de chartes déontologiques pour les professionnels met en exergue des questionnements de nature éthique pour la professionnalisation des métiers de la communication des organisations publiques. 
Au-delà de répondre à une seule logique de légitimation des métiers de la communication publique, ces principes déontologiques concrétisent des questionnements éthiques sur les pratiques et les finalités de l'exercice professionnel. Ainsi, la professionnalisation des métiers de la communication publique, renforcée par l'existence de chartes déontologiques, permet de constater une évolution dans la conception du rôle des professionnels de la communication institutionnelle publique dans la société contemporaine, avec l'affirmation d'une ambition éthique.

\section{Conclusion}

Derrière la quête de légitimité professionnelle, un groupe professionnel contribue à organiser un champ de pratiques mettant en scène les attendus d'un bon professionnalisme (déontologie, échanges entre pairs, éléments de théorisation...), proposant ainsi une régulation de l'exercice de la profession. Les associations de professionnels agissent aussi à d'autres échelles, vis-à-vis des pouvoirs publics et économiques, vis-à-vis des champs de la recherche universitaire. De sorte que des interactions s'opèrent et se croisent entre différents champs sociaux à des niveaux d'organisations, locaux, nationaux. Ainsi, les modalités de leurs actions de représentation et de légitimation se réalisent dans un entre-soi professionnel, dans leurs actions d'unification des pratiques ou champ professionnels, dans leurs opérations de légitimation auprès de pouvoirs établis, qu'ils soient politiques ou institutionnels. Nous avons montré que les revendications de reconnaissance de la part des professionnels connaissent des définitions et des acteurs variables dans le temps mais qui se renforcent continuellement depuis les années 1980 (Bessières 2012). La temporalité des changements sociaux, avec des échelles de temps relativement longues, a des incidences professionnelles. A cela s'ajoute le succès même de l'appellation "communication publique » qui repose beaucoup sur son appropriation par les acteurs de la communication institutionnelle publique euxmêmes et par les dirigeants publics. Mais il doit beaucoup aux actions interactionnistes des groupes et acteurs sociaux, comme les associations représentantes du champ professionnel.

L'association professionnelle s'inscrit dans une perspective d'imposition de reconnaissance interactionniste au sens de la sociologie des professions (Dubar, Tripier, Boussard, 2015) afin de préserver ou d'améliorer la position sociale du groupe socio-professionnel. En cela, elle est éminemment contingente. Elle implique des formes de collaboration et de coordination afin de consolider un équilibre au départ précaire mais qui se solidifie dans le temps. Construction sociale, elle vise à établir les conditions d'une reconnaissance d'un professionnalisme particulier liée à la spécificité publique (conditionnement juridique fort, enjeux politiques prégnants) avec des actions collectives produites par des acteurs du champ professionnel. Les 
regroupements sous formes d'associations professionnelles agissent en participant à la construction d'une représentation globale du groupe, en affirmant une définition. "En effet, un modèle est le produit d'expériences, d'observations, de réflexions sur le réel... Bref, il est un mode d'organisation de la réalité, qui oriente les représentations et les conduites sociales" (Walter 1995, p.13). "Le groupe doit se faire représenter en se donnant des instances officielles et des porte-parole habilités à parler et à agir en son nom » (Boltanski 1982, p.58). Elles fonctionnent comme des supports d'objectivation en donnant à voir, comme une réalité tangible, le champ des professionnels qu'elles promeuvent. Ces visions unificatrices sont de nature interactionniste, portées par des associations.

L'effet de réalité de ces opérations symboliques a été bien décrit par Luc Boltanski (1982) : L'affirmation de ses caractéristiques, vis-à-vis de lui-même et des autres, doit «donner des accentuations dramaturgiques de ses traits pertinents, sorte de stylisation qui contribue à la formation de la croyance collective sans laquelle le groupe n'a pas droit à la reconnaissance sociale». Les principes et chartes déontologiques et éthiques, chartes et manifestes de la communication publique en sont des exemples caractéristiques.

\section{Bibliographie}

Bessières D. (2016). Communication publique et association professionnelle : légitimation et normalisation, Revue Française des sciences d l'information et de la communication, $\mathrm{n}^{\circ}$, , septembre (http://rfsic.revues.org/2003)

Bessières D. (2012). L'institutionnalisation des communicateurs publics : une tension longitudinale vers une légitimation de ce groupe professionnel, revue Pyramides, n²4 (Université Libre de Bruxelles, Belgique), n², 239-260.

Bessières D. (2010). La professionnalisation de la communication publique : des normes de la légitimation du métier à la constitution d'identité disciplinaire. XVIIe Congrès de la Société des Sciences de l'Information et de la Communication : "Au cour et aux lisières des SIC» Dijon, 23-25 juin 2010, (https://www.sfsic.org/index.php/evenements-de-la-sfsic/evenementspasses/congres-2010/17eme-congres-actes-en-ligne)

Bessières, D. (2009). La définition de la communication publique : des enjeux disciplinaires aux changements de paradigmes organisationnels, Communication \& organisation, $\mathrm{n}^{\circ} 35,15-28$.

Bessières D., (2009). La quête de professionnalisation des communicateurs publics entre difficulté et stratégie, in dossier Les processus de professionnalisations, Formation Emploi, $\mathrm{n}^{\circ} 108$ octobre, décembre, 39-52. 
Bessières D., (2008). Suivre le modèle des professions pour légitimer la communication publique : entre lien social interactionniste et être en société par légitimation étatique? Colloque international, AISLF, Istanbul, Turquie, GT01 Savoir, Travail, Profession, 7-11 juillet 2008

Bessières D. (2007). L'institutionnalisation du groupe des communicateurs publics : un chemin critique?, Colloque international Autour de Maurice Hallbacks : classes sociales, groupes sociaux, groupes professionnels, Université de Reims Champagne-Ardenne, 25/ 26 octobre 2007

Bessières D. (1998). L'institutionnalisation de la communication locale : le cas des échelons décentralisés départementaux, régionaux, parisiens franciliens. Thèse de science politique, Université Paris I Panthéon Sorbonne, 541p.

Bouillon J-C., Bourdin S., Loneux C., (2008). Approches communicationnelles des organisations : interroger l'organisation par la communication, Sciences de la société, $\mathrm{n}^{\circ} 74$, mai, 3-9.

Bourdieu P. (1982). Ce que parler veut dire - L'économie des échanges linguistiques, Paris, Fayard, 248 p.

Champagne P. (1990). Faire l'opinion, Paris, Édition de minuit, coll. Le sens commun.

Chapoulie J.-M. (1973). Sur l'analyse sociologique des groupes professionnels, Revue Française de Sociologie, vol. XIV, ${ }^{\circ} 1,86-114$

Dubar C., Tripier P., Boussard V. (2015). Sociologie des professions, 4e Ed, Armand Colin, coll. U, 384 p.

Duran P. (1993), Piloter l'action publique, avec ou sans le droit. Revue Politique et Management Public, vol. 11, n²4, décembre, 1-45.

Fourdin M. (1994). La professionnalisation de la communication locale : un paradoxe ?, Réseaux, $\mathrm{n}^{\circ}$ 64, mars-avril, 75-89.

Gadéa C., (2003). Les cadres en France, Paris, Belin, coll. Perspectives sociologiques.

Habib L. (2010). La communication transformative pour en finir avec les idées vaines, Paris, PUF, 2010, 200 p.

Laufer R., Paradeise C. (1982). Le prince bureaucrate. Paris, Flammarion, 353 p.

Le Saëc O. (2012). Le métier de communicant public et la démocratisation de l'action publique, Communication \& organisation, $\mathrm{n}^{\circ} 41,41-50$.

Loneux C. (2016). La Responsabilité Sociale des Entreprises comme Soft Law: Formes et enjeux de régulation, de dialogue et de frontières, Revue Française 
72 Professionnalisation et éthique de la communication

des sciences $d$ l'information et de la communication, $\mathrm{n}^{\circ} 9$, septembre (http://rfsic.revues.org/2284)

Miège B. (1989). La société conquise par la communication. Grenoble, PUG, 226 p.

Neveu E. (2006). Une société de communication? Paris, Montchrestien, coll. clefs politique, $160 \mathrm{p}$.

Pailliart I. (1993). Les territoires de la communication, Grenoble, PUG, 279 p.

Sfez L. (1990). Critique de la communication, Paris, Le Seuil, 1990

Walter J. (1995). Directeur de communication - Les avatars d'un modèle professionnel, Paris, L'Harmattan, coll. Logiques Sociales, 239 p.

Zémor P. (2008). La communication publique. Paris, PUF, coll. Que sais-je ?, 128 p. 